\title{
Diagnosi e trattamento del rigetto acuto cellulo-mediato nel trapianto di rene
}

\author{
Aris Tsalouchos ${ }^{1}$, Maurizio Salvadori \\ ${ }^{1}$ Azienda USL Toscana Centro, SOS Nefrologia e Dialisi, Ospedale SS. Cosma e Damiano, Pescia, Pistoia - Italia \\ ${ }^{2}$ Professore di Nefrologia già Direttore di Nefrologia e Trapianto, Azienda Ospedaliero-Universitaria Careggi, Firenze - Italia
}

\begin{abstract}
Diagnosis and treatment of acute cell-mediated rejection in kidney transplantation
The incidence of acute rejection of the kidney allograft in the world has been around $15 \%$ during the period between 2001 and 2003. It is clinically defined as an elevation in the level of serum creatinine by more than $0.3 \mathrm{mg} / \mathrm{dL}$ and is diagnosed by kidney biopsy. On pathologic examination, the interstitium of the allograft is diffusely edematous and infiltrated by CD4 and CD8 lymphocytes. Tubulitis occurs when the lymphocytes and monocytes extend into the walls and lumina of the tubules. Presence of leukocytes determines infection or antibody-mediated rejection. Typically C4d staining is negative. Other causes of acute allograft dysfunction included prerenal factors, interstitial nephritis, infection, acute tubular necrosis, toxicity by drugs, and obstruction in the urinary tract. The primary diagnostic assessments include history, especially adherence to immunosuppressive therapy, physical examination, blood and urine laboratory tests, measurement of the serum levels of the drugs, and ultrasonography. Diagnosis of acute cellular rejection depends on biopsy, CD20 staining for refractory cases, negative C4d staining, presence of markers of activating lymphocyte, and proteomic study. Treatment of acute cellular rejection in kidney transplant recipients include pulse steroid for the first rejection episode. It can be repeated for recurrent or resistant rejection. Thymoglobulin and OKT3 are used as the second line of treatment if graft function is deteriorating. Changing the protocol from cyclosporine to tacrolimus or adding mycophenolate mofetil or sirolimus might be effective. Prognosis depends on number of rejection episodes, the use of potent drugs, time of rejection from transplantation, and response to treatment.
\end{abstract}

Keywords: ATG, Diagnosis of acute rejection, Methylprednisolone, Pathology of acute rejection, T cell in acute rejection, Treatment of acute rejection

\section{Introduzione}

Il rigetto acuto cellulo-mediato o rigetto mediato dalle cellule T [T Cell-Mediated (cellular) Rejection, TCMR] è definito clinicamente come un declino della funzione renale del graft e diagnosticato da specifiche lesioni patologiche identificate con la biopsia renale.

Secondo i criteri di classificazione istologica della Banff Foundation for Allograft Pathology, il TCMR può essere caratterizzato da lesioni acute (TCMR-acuto), lesioni sospette (borderline) per rigetto acuto (TCMR-borderline) e lesioni croniche attive (TCMR-cronico attivo) (Tab. I) (1). L'evidenza istologica

Received: November 27, 2019

Accepted: December 31, 2019

Published online: February 27, 2020

Indirizzo per la corrispondenza:

Maurizio Salvadori

Azienda Ospedaliero-Universitaria Careggi

Largo Brambilla, 3

50134 Firenze - Italia

maurizio.salvadori1@gmail.com di TCMR-acuto senza aumento della concentrazione sierica di creatinina è nota come rigetto subclinico.

Recentemente, sono stati identificati dei biomarcatori sierici e urinari, e quindi non invasivi, che possono individuare pazienti ad alto rischio di sviluppare rigetto acuto e con rigetto acuto in atto (2). Il vantaggio dell'utilizzo di questi biomarcatori nella pratica clinica è quello di prevedere il rigetto acuto in modo tale da potenziare preventivamente la terapia immunosoppressiva nei pazienti ad alto rischio e in più diagnosticare in modo precoce il rigetto, soprattutto quello subclinico, per impostare prima possibile il trattamento adeguato. Tuttavia, la limitazione dei biomarcatori non invasivi è quella di non riuscire a diversificare il TCMR dal rigetto anticorpo-mediato (Antibody-Mediated Rejection, AMR). Moderne tecniche di imaging funzionale sono inoltre promettenti nella diagnosi differenziale di rigetto acuto da altre cause di disfunzione del graft (3).

L'utilizzo degli attuali protocolli di terapia immunosoppressiva, di induzione e mantenimento, ha ridotto sensibilmente l'incidenza del TCMR (10-15\%) che però continua a rappresentare una delle principali cause di disfunzione del graft a lungo termine, anche se non tutti gli episodi di rigetto 
hanno lo stesso impatto (4). La maggiore gravità istologica e la presenza di episodi di rigetto tardivo (>3mesi post-trapianto) sono associate a risultati peggiori.

Nella prima parte di questa review riportiamo le manifestazioni cliniche di rigetto acuto, le caratteristiche radiologiche, i criteri istologici di diagnosi per il TCMR e i nuovi biomarcatori per il rigetto, mentre nella seconda parte discutiamo l'approccio terapeutico nei pazienti ad alto rischio di TCMR e il trattamento del TCMR sulla base della severità istologica.

\section{Diagnosi}

La diagnosi di rigetto acuto richiede un processo graduale che inizia con manifestazioni cliniche sospette e non di assoluta certezza, continua con l'esclusione di cause secondarie di disfunzione del graft e si conclude con l'esecuzione della biopsia renale che rappresenta il gold-standard per la diagnosi (Fig. 1).

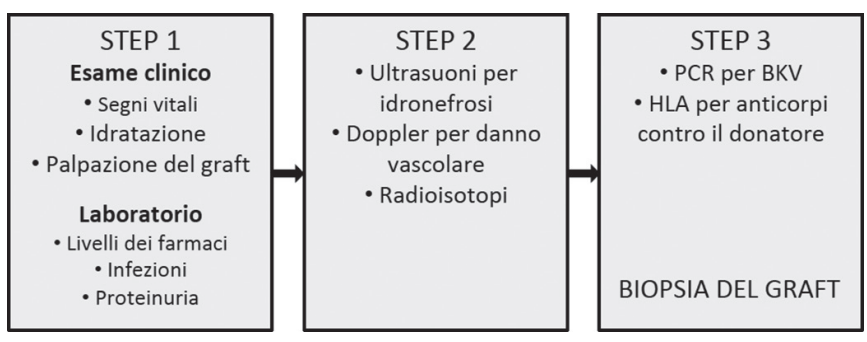

Fig. 1 - Step diagnostici per rigetto acuto

\section{Manifestazioni cliniche di rigetto acuto}

Gli episodi di TCMR si verificano più comunemente entro il primo anno dopo il trapianto ed episodi di rigetto dopo questo periodo sono tipicamente dovuti alla mancata aderenza del paziente alla terapia immunosoppressiva di mantenimento o alla eccessiva riduzione della stessa.

Le manifestazioni cliniche del rigetto acuto sono comuni per il TCMR e I'AMR (5). Nella pratica clinica, il sospetto di rigetto acuto si pone nei pazienti che si presentano con disfunzione del graft caratterizzata dall'aumento dei valori di creatinina sierica ( $\geq 0.3 \mathrm{mg} / \mathrm{dL}$ rispetto al valore abituale), confermato con misurazioni seriate. In caso di rigetto, la disfunzione del graft è comunemente associata con la comparsa o con l'aumento del grado della proteinuria preesistente. Inoltre, il sospetto di rigetto acuto insorge nei pazienti con ritardata ripresa di funzionalità del graft (Delayed Graft Function, DGF), definita con la richiesta di dialisi nella prima settimana dopo il trapianto, che si prolunga oltre questo periodo.

La maggioranza dei pazienti con rigetto acuto è asintomatica con gli attuali regimi terapeutici. Tuttavia, manifestazioni cliniche simil-influenzali (febbricola, astenia, malessere generale), contrazione della diuresi con comparsa di edemi declivi e dolenzia in fossa iliaca nella sede del trapianto e/o tumefazione del graft, possono accompagnare la disfunzione del graft nei casi più gravi di rigetto.

La diagnosi differenziale con altre cause di disfunzione del graft è d'obbligo al momento della presentazione prima di eseguire la biopsia renale (Tab. I).

Tabella I - Diagnosi differenziale di disfunzione dell'organo

\begin{tabular}{|l|}
\hline Prima settimana dopo il trapianto \\
\hline Necrosi tubulare acuta \\
\hline Rigetto iperacuto o accelerato \\
\hline Complicanze urologiche \\
\hline$\bullet \quad$ Ostruzione \\
\hline$\bullet \quad$ Deiscenza urinaria \\
\hline Trombosi vascolare \\
\hline$\bullet \quad$ Arteriosa \\
\hline Venosa \\
\hline Ipovolemia \\
\hline Primo anno dopo il trapianto \\
\hline Rigetto acuto \\
\hline Tossicità da inibitori della calcineurina \\
\hline Ipovolemia \\
\hline Complicanze urologiche \\
\hline \multicolumn{1}{c}{ Ostruzione } \\
\hline Infezioni \\
\hline • \\
\hline Pielonefrite batterica \\
\hline Infezioni virali \\
\hline Nefrite interstiziale \\
\hline Recidiva di malattia renale \\
\hline Dopo il primo anno \\
\hline Rigetto acuto \\
\hline Ipovolemia \\
\hline Tossicità da inibitori della calcineurina \\
\hline Complicanze urologiche \\
\hline Recidiva di malattia renale \\
\hline Infezioni \\
\hline \\
\hline
\end{tabular}

\section{Tecniche di imaging nella diagnosi di rigetto acuto}

L'utilizzo della diagnostica per immagini è utile in primis per escludere cause secondarie di disfunzione del graft. Inoltre, può potenziare il sospetto di rigetto con l'utilizzo delle più recenti tecniche di acquisizione funzionale del graft (3).

In caso di rigetto acuto, all'esame ecografico il graft può apparire di dimensioni aumentate a causa dell'edema e 
ipoecogeno con perdita della differenziazione corticomidollaree prominenza delle piramidi, mentre gli indici di resistenza (IR) intraparenchimali possono essere aumentati (>0.80). Tuttavia, questo pattern ecografico può essere sovrapponibile a quanto osservato in corso di Necrosi Tubulare Acuta (NTA) e, pertanto, aspecifico di rigetto acuto (6).

Lo studio mediante ecografia con mezzo di contrasto (Contrast-Enhanced Ultrasound, CEUS) potrebbe dare delle informazioni utili sulla micro perfusione renale nettamente superiori alla stima della vascolarizzazione mediante color- e power-Doppler. In pazienti con rigetto acuto la CEUS può evidenziare un aspecifico ritardo nell'enhancement della corticale (6).

Grabner et al. (7) hanno mostrato, su modello animale, che un rilevamento più specifico di rigetto acuto con la CEUS è possibile marcando le microbolle con anticorpi in grado di riconoscere epitopi presenti sulla superficie cellulare di linfociti $T$ che infiltrano il parenchima del graft in corso di TCMR. In questo modo l'ecogenicità del graft può essere sufficientemente aumentata per consentire una diagnosi affidabile di TCMR.

La risonanza magnetica (RM) funzionale è una metodica di recente introduzione nel campo dell'imaging che dà informazioni aggiuntive rispetto alla semplice morfologia senza utilizzare alcun mezzo di contrasto esogeno (8). A differenza dell'imaging di RM pesata in diffusione (Diffusion Weighted Imaging, DWI), poco specifica per il rigetto acuto, la Blood Oxygen Level Dependent (BOLD) RM sembra in grado di distinguere il rigetto acuto dalla NTA. La BOLD RM permette di misurare in modo indiretto e non invasivo lo stato d'ossigenazione del parenchima renale, sfruttando le proprietà paramagnetiche della desossiemoglobina. Studi preliminari hanno evidenziato che nei pazienti con rigetto acuto la midollare renale mostra maggiore ossigenazione rispetto alla midollare dei pazienti con NTA e alla midollare del rene trapiantato normale. Le due ipotesi proposte per questa differenza sono una riduzione dell'utilizzo dell'ossigeno e/o un incremento degli shunts cortico-midollari.

La glucose Chemical Exchange Saturation Transfer (glucoCEST) RM è una nuova metodica che utilizza come mezzo di contrasto il D-glucosio.

\section{Patologia}

La già citata classificazione di Banff (1) classifica il rigetto acuto cellulo-mediato come è evidenziato nella Tabella II.

Per comprendere meglio la terminologia si deve intendere per tubulite $(t)$ la presenza di cellule mononucleate nello spazio basolaterale dell'epitelio tubulare renale. A seconda dell'intensità delle lesioni, la tubulite può andare dal grado 0 al grado 3 . L'infiammazione interstiziale (i), che è un marker frequente del TCMR, è rappresentata da segni di flogosi (cellule infiammatorie) in aree corticali non cicatriziali. Nuovamente si va dal grado 0 al grado 3 a seconda dell'intensità della flogosi nell'area interessata. L'arterite intimale o endoarterite $(\mathrm{v})$ è caratterizzata dalla presenza di cellule
Tabella II - Classificazione di Banff del rigetto acuto TCMR

\begin{tabular}{|l|l|}
\hline Grado I A & $\begin{array}{l}\text { Flogosi interstiziale interessante }>25 \% \text { della corticale } \\
\text { non sclerotica (i2 o i3) con tubulite moderata (t2) in- } \\
\text { teressante 1 o più tubuli }\end{array}$ \\
\hline Grado I B & $\begin{array}{l}\text { Flogosi interstiziale interessante }>25 \% \text { della corticale } \\
\text { non sclerotica (i2 o i3) con grave tubulite (i3) interes- } \\
\text { sante 1 o più tubuli }\end{array}$ \\
\hline Grado II A & $\begin{array}{l}\text { Arterite lieve o moderata (v1) con o senza flogosi in- } \\
\text { terstiziale e/o tubulite }\end{array}$ \\
\hline Grado II B & $\begin{array}{l}\text { Grave arterite intimale (v2) con o senza flogosi inter- } \\
\text { stiziale e/o tubulite }\end{array}$ \\
\hline Grado III & $\begin{array}{l}\text { Arterite trans-murale e/o necrosi fibrinosi vascolare } \\
\text { con arterite intimale con cellule mononucleate (v3), } \\
\text { con o senza flogosi interstiziale e/o tubulite }\end{array}$ \\
\hline
\end{tabular}

$\mathrm{I}=$ infiammazione interstiziale; $\mathrm{t}=$ tubulite $\mathrm{v}=$ arterite intimale $\mathrm{o}$ endoarterite

infiammatorie, soprattutto linfociti e monociti nello spazio sub-endoteliale di una o più arterie. Si va nuovamente dal grado 0 al grado 3 . Nel grado 3 si ha la cosiddetta arterite trans-murale con necrosi delle cellule muscolari lisce e infiltrazione di linfociti nei vasi (9).

\section{Biomarkers}

Con tutti i dubbi sopra riportati esistono molecole che rappresentano biomarkers di rigetto acuto (TCMR). Sono sostanze che, qualora presenti nel ricevente, sono suggestive per la presenza di rigetto TCMR.

Esistono biomarkers nel pre-trapianto e biomarkers nella fase di rigetto acuto. I primi sono solo predittivi, i secondi hanno valore diagnostico. I biomarcatori diagnostici per TCMR e riportati in studi di proteomica sono evidenziati nella Tabella III. Si tratta di proteine presenti o nel sangue o nelle urine, frequentemente associate a TCMR. Tuttavia, la biopsia renale rimane ancora il gold standard diagnostico.

\section{Terapia}

In caso di TCMR, la biopsia renale è fondamentale perché la gravità istopatologica guida la scelta terapeutica.

II rigetto borderline è rappresentato da un rigetto TCMR con lesioni molto modeste, trovato incidentalmente in biopsie protocollari, in assenza di segni clinici di rigetto. In pazienti con TCMR borderline molti preferiscono non trattare il rigetto. Tuttavia, se i livelli degli inibitori della calcineurina nel siero sono troppo bassi, è consigliato aumentarli fino a raggiungere range terapeutici. II paziente va tuttavia attentamente sorvegliato perché da una situazione borderline non è escluso lo sviluppo di un rigetto acuto clinicamente e istologicamente manifesto.

Nella maggioranza dei pazienti con TCMR grado I A o I B, boli endovena di metilprednisolone da $500 \mathrm{mg}$, ripetuti fino a 3-5 volte, sono in grado di controllare il rigetto. È spesso opportuno aumentare le dosi dell'immunosoppressione di mantenimento. 
Tabella III - Biomarkers di proteomica per rigetto acuto

\begin{tabular}{|c|c|c|c|c|}
\hline Autore & Biomarker candidato & Fluido esaminato & Campioni & Esito valutato \\
\hline Freue et al. (12) & $\begin{array}{l}\text { TIN, LBP, CFD, MBL2, SERINA 10, AFM, KNG1, LCAT, SHBG, } \\
\text { UMOD, PEDF, CD } 44\end{array}$ & Plasma & 32 & AR \\
\hline Sigdel et al. (13 & UMOD, PEDF, CD44 & Urine & 60 & $A R$ \\
\hline Wu et al. (14) & NF-kB, STAT1, STAT3 ed altre 63 proteine & Plasma & 13 & $A R$ \\
\hline Loftheim et al. (15) & IGFB7, VASN, EGF, LG3BP & Urine & 12 & AR \\
\hline Sigdel et al. (16) & HLA-DRB1, FGB, FGA, KRT14, HIST1H4B, ACTB, KRI7, DPP4 & Urine & 154 & $A R$ \\
\hline
\end{tabular}

$A R=$ rigetto acuto; $T I N=$ titin $L B P=$ proteina legata ai lipidi; $C F D=; M B L 2=$ mannoso legato alla lectina $2 ;$ SERINA 10 = Inibitore protesico dipendente dalla proteina Z; AFM = forza atomica microscopica; KNG1 = kininogeno 1; LCAT = lecitina colesterolo aciltransferasi; SHBG = proteina legata all'ormone sessuale; UMOD = uromodulina; PEDF = pigmento derivato dal fattore epiteliale; CD44 =; NF-kB = fattore nucleare kB; STAT1 = segnale di trasduzione e attivazione 1; STAT3 = segnale di trasduzione e attivazione 3; IGFB7 = fattore di crescita simil-insulinico 7; VASN = vasorina; EGF = fattore di crescita epidermico; $L$ G3BP = proteina legante la galattina; HLA-DRB1 =; FGB = precursore del fibrinogeno $\beta ; F G A=$ precursore del fibrinogeno $\alpha ; \mathrm{KRT} 14=$ keratina $14 ; \mathrm{HIST} 1 \mathrm{H} 4 \mathrm{~B}=$ membro della famiglia B dell'istone $1 \mathrm{H} 4 ; \mathrm{ACTB}=$ actina $\beta ; \mathrm{KRT7}=$ keratina 7 ; DPP4 = peptidasi di peptidica 4

Nei pazienti che presentino un TCMR acuto di grado I B senza lesioni croniche e nel primo periodo post-trapianto, molti preferiscono somministrare globuline antitimocitarie ( $r A T G)$ in aggiunta ai boli di steroide (10). II razionale di un trattamento aggressivo risiede nel fatto che organi affetti da tali rigetti vanno più frequentemente incontro a outcomes peggiori.

In pazienti con rigetto di grado II o III, in aggiunta ai boli di metilprednisolone vengono spesso associate rATG a dosi singole da 1,5 o $3 \mathrm{mg} / \mathrm{kg}$ fino ad arrivare a dosi totali di 5-10 $\mathrm{mg} / \mathrm{kg}$. Le dosi sono anche aumentate nel caso di rigetti di grado III.

Esistono pazienti che non possono ricevere trattamento con rATG perché allergizzati alle proteine di coniglio. Questo accade il più delle volte a pazienti già trattati in precedenza

\section{Bibliografia}

1. Roufosse C, Simmonds N, Clahsen-van Groningen $M$, et al. A 2018 Reference Guide to the Banff Classification of Renal Allograft Pathology. Transplantation. 2018;102(11):1795-814.

2. Salvadori M, Tsalouchos A. Biomarkers in renal transplantation: An updated review. World J Transplant. 2017;7(3):161-78.

3. Köhnke R, Kentrup D, Schütte-Nütgen $K$, et al. Update on imaging-based diagnosis of acute renal allograft rejection. Am J Nucl Med Mol Imaging. 2019;9(2):110-26.

4. Tanriover B, Jaikaransingh V, MacConmara MP, et al. Acute Rejection Rates and Graft Outcomes According to Induction Regimen among Recipients of Kidneys from Deceased Donors Treated with Tacrolimus and Mycophenolate. Clin J Am Soc Nephrol. 2016;11(9):1650-61.

5. Brennan DC, AlhamadT, MaloneA. Clinical features and diagnosis of acute renal allograft rejection. https://www.uptodate. com/contents/clinical-features-and-diagnosis-of-acute-renalallograft-rejection?source=bookmarks_widget\#H792385721. Accessed August 25, 2019.

6. Jehn U, Schuette-Nuetgen K, Kentrup D, Hoerr V, Reuter S. Renal Allograft Rejection: Non invasive Ultrasound- and MRI-Based Diagnostics. Contrast Media Mol Imaging. $2019 \mathrm{Apr}$ 10;2019:3568067.

7. Grabner A, Kentrup D, Mühlmeister $M$, et al. Noninvasive imaging of acute renal allograft rejection by ultrasound detection of microbubbles targeted to T-lymphocytes in rats. Ultraschall Med. 2016;37(1):82-91.

8. van Eijs MJM, van Zuilen $A D$, de Boer $A$, et al. Innovative perspective: gadolinium-free magnetic resonance imaging con rATG. Tali pazienti possono facilmente andare incontro a malattia da siero. In tali pazienti al posto delle rATG è possibile somministrare un singolo bolo endovenoso di $30 \mathrm{mg}$ di alemtuzumab (11).

In tutti i pazienti trattati con rATG o alemtuzumab si raccomanda di eseguire una profilassi antimicrobica e antivirale. Particolare attenzione va posta alla possibile insorgenza di infezioni da Pneumocystis pneumoniae, Citomegalovirus e virus erpetici.

\section{Disclosures}

Financial support: The Authors declare that no financial support was received for this submission.

Conflict of interest: The Authors declare no conflict of interest. in long-term follow-up after kidney transplantation. Front Physiol. 2017;8:296.

9. Loupy A, Haas M, Solez K, et al. The Banff 2015 Kidney meeting report: current challanges in rejection classification and prospects for adopting molecular pathology. Am J Transplant. 2017;17(17):28-41.

10. Minervini MI, Torbenson $\mathrm{M}$, Scantlebury $\mathrm{V}$, et al. Acute renal allograft rejection with severe tubulitis (Banff 1997 grade IB). Am J Surg Pathol. 2000;24(4): 553-8.

11. Boothpur R, Hardinger KL. Skelton RM, et al. Serum sickness after treatment with rabbit antithymocyte globulin in kidney transplant recipients with previous rabbit exposure. Am J Kidney Dis. 2010;55:141-3.

12. Freue GV, Sasaki M, Meredith A, et al. Proteomic signatures in plasma during early acute renal allograft rejection. Mol Cell Proteomics. 2010;9(9):1954-1967.

13. Sigdel TK, Kaushal A, Gritsenko M, et al. Shotgun proteomics identifies proteins specific for acute renal transplant rejection. Proteomics Clin Appl. 2010;4(1):32-47.

14. Wu D, Zhu D, Xu M, et al. Analysis of transcriptional factors and regulation networks in patients with acute renal allograft rejection. J Proteom Res. 2011;10: 175-181.

15. Loftheim $\mathrm{H}$, Midtvedt $\mathrm{K}$, Hartmann A, et al. Urinary proteomic shotgun approach for identification of potential acute rejection biomarkers in renal transplant recipients. Transplant Res. 2012;31;1(1):9.

16. Sigdel TK, Salomonis N, Nicora CD, et al. The identification of novel potential injury mechanisms and candidate biomarkers in renal allograft rejection by quantitative proteomics. Mol Cell Proteomics. 2014;13(2):621-631. 